\title{
Variáveis de Impacto na Qualidade de Vida de Pessoas Acima de 50 Anos HIV+
}

\author{
Variables of Impact on Quality of Life of HIV-Positive People Over 50 Years Old
}

\author{
Josevânia da Silva*, Ana Alayde Werba Saldanha \& Regina Lígia Wanderlei de Azevedo \\ Universidade Federal da Paraíba
}

\begin{abstract}
Resumo
Este estudo objetivou analisar a influência de variáveis bio-demográficas e clínicas na qualidade de vida (QV) de pessoas acima de 50 anos HIV+. Para tanto, contou-se com a participação de 43 pessoas HIV+ acima de 50 anos $(M=55 ; D P=4,6)$, sendo $63 \%$ do sexo masculino. Utilizou-se como instrumentos a Escala de Qualidade de Vida para a Velhice (WHOQOL-Old) e um questionário bio-demográfico. Verificouse o impacto de variáveis bio-demográficas e clínicas nos seguintes fatores: "Autonomia", "Atividades passadas, presentes e futuras", "Morte e Morrer" e "Intimidade". A QV apresentou-se como uma dimensão da vida humana indissociável de condições objetivas como trabalho, lazer, moradia, dentre outros. Além disso, esse construto está relacionado a características subjetivas, próprias de cada indivíduo, como o suporte social.
\end{abstract}

Palavras-chave: Velhice; Qualidade de vida; AIDS; Biodemográfico.

\begin{abstract}
This study aimed to analyze the influence of biodemographic and clinical variables on quality of life of HIV-positive people over 50 years old. For that, 43 HIV-positive people over 50 took part in the study $(M=55 ; D P=4.6)$, being $63 \%$ male. The Scale of Quality of Life in Old Age (WHOQOL-OLD) and a biodemographic questionnaire were used as instruments. It was verified the impact of biodemographic and clinical variables in the following factors: "Autonomy", "Past, Present and Future Activities", "Death and Dying" and "Intimacy". The QL presented itself as a dimension of human life which is inseparable from objective conditions such as work, leisure, and housing, among others. Moreover, it is related to subjective characteristics, specific to each individual, such as social support.

Keywords: Old age; Quality of life; AIDS; Biodemographic.
\end{abstract}

O construto qualidade de vida vem, desde os anos 60 , despertando interesse na literatura psicológica e de saúde pública em geral, e vem sendo utilizada de forma cada vez mais frequente (Canavarro, Simões, Pereira, \& Pintassilgo, 2005). Na área da saúde, o interesse pela qualidade de vida decorre, sobretudo, da definição de saúde assumida pela Organização Mundial de Saúde ([OMS], 1994), como um estado de completo bem-estar físico, mental e social e não simplesmente como a ausência de doença. Assim, a qualidade de vida relacionada com a saúde é um conceito que tem como enfoque a avaliação subjetiva do paciente e está, necessariamente, relacionado ao impacto do estado de saúde sobre a capacidade do indivíduo de viver plenamente. Busca-se avaliar a maneira pela qual a vida do indivíduo é afetada por uma doença ou por vários componentes de seu tratamento. No entanto, deve-se considerar que se trata de

${ }^{*}$ Endereço para correspondência: Universidade Federal da Paraíba, Centro de Ciências Humanas, Letras e Artes, Campus I, Jardim Cidade Universitária, João Pessoa, PB, Brasil, CEP 58051-000.E-mail: josevaniasco@gmail.com um termo amplo e inclui uma variedade de condições que podem afetar a percepção do indivíduo, seus sentimentos e comportamentos relacionados com o seu funcionamento diário, incluindo a sua condição de saúde e as intervenções médicas, sem, no entanto, limitar-se a elas (Fleck et al., 1999).

Considerando-se a dimensão subjetiva do construto na avaliação da qualidade de vida, faz-se necessário enfatizar os atributos da vida valorizados pelos pacientes, tais como: conforto, senso de bem-estar, funcionamento físico, emocional e intelectual e a habilidade para participar de atividades. Desse modo, busca-se avaliar a experiência da doença, levando-se em conta não apenas medidas objetivas de gravidade da doença, mas também a percepção do paciente acerca dos sintomas, a maneira como ele os comunica a outras pessoas, a experiência de não ser capaz de desempenhar suas atividades e as estratégias de enfrentamento no lidar com a doença.

Ainda nesse enfoque, características individuais podem interferir na qualidade de vida, sejam elas de natureza biológica, social e econômica como, por exemplo, variáveis como sexo, faixa etária, situação conjugal, situação ocupacional, escolaridade, renda per capita, tempo 
de infecção (tempo de convívio com a doença desde seu diagnóstico) e tempo de tratamento, ou seja, tempo de uso da medicação recomendada para o tratamento da patologia (Arpinelli, Visona, Bruno, Carli, \& Apolone, 2000; Avis, Smith, \& Swislow, 1997; Badia, Guerra, Garcia, \& Podzamczer, 1999; Burgoyne \& Saunders, 2001). No tocante à variável faixa etária, verifica-se que a população acima de 50 anos tem sido foco de pesquisas (Araújo, Coutinho, \& Saldanha, 2005), mais especificamente de estudos que avaliam a qualidade de vida, haja vista o aumento na expectativa de vida da população mundial, bem como o aumento do número de casos de pessoas soropositivas ao HIV (Vírus da Imunodeficiência Humana) nessa faixa etária. Entre idosos, aspectos como gênero, aposentadoria e escassez de recursos econômicos podem afetar a qualidade vida.

$\mathrm{Na}$ literatura, verificam-se casos de pessoas acima de 50 anos apresentando algum tipo de doença crônica, dentre algumas a AIDS (Síndrome da Imunodeficiência Adquirida). Desde o advento da AIDS até 1997, o Brasil possuía cerca de 7.239 casos dessa doença referentes às pessoas na faixa etária de 50 a 59, e 2.835, com idades igual ou superior a 60 anos. Passados dez anos, o número de pessoas com AIDS, nessas faixas etárias, passou para 32.231 e 11.110 casos, respectivamente. Assim, o número de pessoas com AIDS, com idade igual ou superior a 50 anos, passou de 10.074, em 1997, para 43.341 em 2007 (Ministério da Saúde, 2007).

As estimativas de suscetibilidade por idade têm demonstrado que o "risco relativo de infecção pelo HIV" apresenta crescimento a partir da faixa etária de 13 anos, atinge o máximo após os 20 anos, diminuindo até os 40 anos, recomeçando o crescimento após essa idade (M. T. S. Barbosa \& Struchiner, 2003). Tais índices indicam a necessidade de campanhas de prevenção e a inserção desse segmento populacional em ensaios clínicos, bem como a promoção de intervenções educativas voltadas para as pessoas acima de 50 anos de idade e para seus cuidadores formais e informais. Todavia, de acordo com Saldanha e Araújo (2006), a infecção pelo HIV/AIDS nessa faixa etária não é, frequentemente, diagnosticada de forma precoce.

A falsa crença de alguns profissionais de saúde de que a AIDS, dificilmente, ocorrerá nessa fase da vida decorre, em parte, do "mito" da inexistência de uma vida sexualmente ativa nessa população. No entanto, a utilização de terapias hormonais e a descoberta de novos fármacos têm contribuído de forma significativa para uma melhoria da atividade sexual nesse segmento (Dias, Fonseca, Renca, \& Silva, 2005), configurando-se na principal via de contaminação da AIDS entre os idosos (Cloud, Browne, Salooja, \& Mclean, 2003). Por outro lado, essa melhora na qualidade da vida sexual na velhice não é acompanhada por igual política de saúde de prevenção das DST`s (Doenças Sexualmente Transmissíveis), bem como de uma melhor compreensão do próprio processo de envelhecimento (Souza \& Leite, 2002).
Contudo, nas últimas décadas, mudanças vêm ocorrendo na prática e no pensamento sobre a sexualidade humana e, consequentemente, sobre a sexualidade na velhice. Para Ribeiro (1997), essas mudanças decorrem da influência de três processos, a saber: (a) a mudança na função da atividade sexual - ou seja, o que antes tinha a função de procriação, agora tem o afeto como elemento norteador de satisfação pessoal dos relacionamentos; (b) o significativo aumento dos anos na expectativa de vida da população mundial com condições psíquica e física satisfatórias e disposta a vivenciar sua sexualidade; (c) o surgimento da AIDS, exigindo, por parte da sociedade, repensar a maneira como lidava com a sexualidade.

Assim, a soropositividade para o HIV/AIDS na maturidade e velhice, de certo modo, revela o exercício da sexualidade nessa população e demonstra que, com o avançar do desenvolvimento humano, as pessoas não se tornam assexuadas, dessa forma são vulneráveis ao risco de contrair DST's (Butin, 2002). Nesse contexto, Ayres, França e Calazans (1997) definiram a vulnerabilidade como graus e naturezas de susceptibilidade de indivíduos e coletividades à infecção, adoecimento e morte pela infecção por HIV, segundo particularidades formadas pelo conjunto dos aspectos sociais, programáticos e individuais que os põem em relação com o problema e com os recursos para seu enfrentamento. Assim, a vulnerabilidade pode expressar-se no plano individual, social e programático/institucional.

Um estudo realizado por Fleck, Chachamovich e Trentini (2006) com 90 idosos internados em um hospital geral universitário, objetivando verificar as variáveis associadas à percepção de saúde, demonstrou, dentre outros resultados, uma prevalência alta de idosos que se percebiam como saudáveis. Esses resultados sugerem a necessidade de se estudar a qualidade de vida na maturidade e velhice, considerando-se o caráter multidimensional do construto. Para alguns autores (Backman, Mantyla, \& Herlitz, 1990; Baltes \& Baltes, 1990), a qualidade de vida na velhice poderia ser entendida como um processo de adaptação com características multidimensionais, que compreenderia a capacidade do sujeito de lidar com demandas recorrentes. Todavia, embora a multidimensionalidade da qualidade de vida na velhice seja consenso na literatura, encontram-se dificuldades em precisar quais seriam as variáveis de maior impacto, uma vez que o envelhecimento se dá de acordo com a historicidade, a cultura, as vivências e as condições materiais de cada indivíduo, não sendo possível falar em uma única velhice, mas em velhices.

Pesquisas desenvolvidas no âmbito da Gerontologia (Jakobsson, Klevsgard, Westergren, \& Hallberg, 2003; Neri, 2001) têm apontado para a importância do funcionamento do sensório e da autonomia como fatores que contribuem para uma qualidade de vida satisfatória na avaliação de idosos. No contexto de pessoas acima de 50 anos, com AIDS, há que se considerar que o impacto 
trazido pela doença produz sofrimento tanto físico quanto psicológico e social. Segundo Saldanha e Araújo (2006), essas pessoas experimentam sentimentos de impotência diante da doença; de culpa e vergonha diante dos outros; sensação de proximidade da morte e falta de preparo para lidar com a situação.

Enquanto fato social, a AIDS, além do sofrimento causado pela doença em si, é potencializada pelo estigma e pelo preconceito, contribuindo para a "morte social" da pessoa. Assim, considerando-se os estados de instabilidade emocional, os episódios depressivos e o sofrimento psíquico vivenciados pelas pessoas soropositivas para o HIV/AIDS, bem como o impacto desses fatores na qualidade de vida, o presente estudo teve como objetivo analisar a influência de variáveis biodemográficas e clínicas na qualidade de vida de pessoas acima de 50 anos soropositivas para o HIV/AIDS.

\section{Método}

\section{Delineamento}

O presente estudo teve um delineamento descritivo e bivariado, tendo como variáveis a Escala de Qualidade de Vida para a velhice (WHOQOL-Old), a pessoa soropositiva para o HIV e os aspectos biodemográficos.

\section{Participantes}

A amostra pesquisada foi composta por 43 pessoas soropositivas para o HIV/AIDS, com idades variando de 51 a 78 anos $(M=55 ; D P=4,6)$, sendo $63 \%$ do sexo masculino; $46 \%$ casados ou conviventes; $30 \%$ com ensino médio completo, $86 \%$ católicos; $74 \%$ com renda até um salário mínimo ( $\mathrm{R} \$ 415,00) ; 51 \%$ residentes no interior da Paraíba; $39 \%$ têm de 3 a 5 anos de tempo de diagnóstico e $48 \%$ já tiveram algum tipo de doença oportunista. A escolha dos participantes se deu de forma não-probabilística e acidental, em um hospital público, referência no atendimento e tratamento de pessoas soropositivas para o HIV, localizado na cidade de João Pessoa/PB, Nordeste do Brasil.

\section{Instrumentos}

Para a coleta dos dados foi utilizado inicialmente um questionário biodemográfico, cujas questões tiveram por finalidade melhor caracterizar os participantes quanto ao sexo, idade, grau de escolaridade, renda familiar (salário mínimo correspondente a $\mathrm{R} \$ 415,00$ no momento da pesquisa), situação conjugal, local de residência, tipo de religião e dados clínicos - como tempo de infecção diagnosticada e de tratamento (tempo de uso da terapia antiretrovial).

Em seguida, aplicou-se a escala WHOQOL-Old, que foi desenvolvida pelo World Health Organization Quality of Life Group (OMS, 2005) em uma perspectiva transcultural para medir qualidade de vida de pessoas na velhice, considerando como características fundamentais o caráter subjetivo do construto (englobando aspectos positivos e negativos), e sua natureza multidimensional.

A escala possui 24 itens, respondidos em uma escala tipo Likert, atribuídos a seis fatores. Cada fator possui 4 itens, a saber: Funcionamento do Sensório (FS); Autonomia (AUT); Atividades Passadas, Presentes e Futuras (PPF); Participação Social (PSO); Morte e Morrer (MEM), e Intimidade (INT), (Fleck et al., 2006; OMS, 2005).

\section{Procedimentos}

Foi realizado contato com o diretor da instituição hospitalar para a apresentação dos objetivos do estudo e a importância do local para o desenvolvimento do mesmo. Após sua autorização e a aprovação do Comitê de Ética em Pesquisa do Centro de Ciências da Saúde da Universidade Federal da Paraíba, foi iniciada a aplicação dos instrumentos. Ao serem contatados, os participantes foram informados acerca do estudo, explicitando o caráter voluntário da sua participação seguido da assinatura do termo de consentimento livre e esclarecido. Por conseguinte, foi solicitado a cada participante respondesse, individualmente, a Escala de Qualidade de Vida e o questionário bio-demográfico.

\section{Análise dos Dados}

Com os dados obtidos através do questionário biodemográfico, foram realizadas análises de estatística descritiva. Já os dados decorrentes da Escala de Qualidade de Vida para Idosos (WHOQOL-Old) foram analisados conforme as instruções para aplicação e avaliação do instrumento fornecido pelo Centro WHOQOL do Brasil. Assim, os escores foram transformados em índices com ponderação de 0 a 100, em que 0 corresponde ao menor e 100 ao maior valor encontrado para cada domínio, considerando-se, portanto, como prejudicados os domínios que apresentaram índices cuja média foi inferior a 50 pontos, uma vez que a pontuação máxima poderia ser 100 pontos. Desse modo, para a identificação da influência das variáveis bio-demográficas e clínicas na qualidade de vida dos participantes $\mathrm{HIV}+$, foram feitas comparações, através do teste $t$, entre as médias dos fatores da Escala de Qualidade de Vida para Idosos e as médias dos grupos critérios das variáveis biodemográficas e clínicas. Tais procedimentos estatísticos foram realizados por meio do Pacote Estatístico para as Ciências Sociais (SPSS), versão 15.0.

\section{Resultados e Discussão}

Embora a maioria dos participantes seja do sexo masculino $(63 \%)$, os resultados indicaram que a proporção de homens infectados para cada mulher é menor que dois, refletindo a tendência epidemiológica da doença no país, ou seja, a "feminização" da AIDS. Além disso, a partir do perfil dos participantes, confirmaram-se os resulta- 
Silva, J., Saldanha, A. A. W. \& Azevedo, R. L. W. (2010). Variáveis de Impacto na Qualidade de Vida de Pessoas Acima de 50 Anos HIV+.

dos de pesquisas anteriores (Bastos \& Szwarcwald, 2000; Cruz, 2005; Saldanha, Figueiredo, \& Coutinho, 2004), em que se dissertou sobre a "pauperização" e a "interiorização" da AIDS, uma vez que no Brasil, ainda que com algumas restrições, a "pauperização" da AIDS é verificada de acordo com a escolaridade das pessoas atingidas pela doença (Brito, Castilho, \& Szwarcwald, 2001).

A relação entre saúde e nível sócio-econômico é mensurada, dentre outros indicadores, através da renda, ocupação e escolaridade, sendo este último um dos indicadores mais importante (Cairney \& Arnold, 1998; Sorlie, Backlund, \& Keller, 1995). Segundo Fonseca et al. (2000), a renda pode indicar estimativas quanto ao acesso de bens materiais e serviços de saúde, já a escolaridade pode diferenciar as pessoas quanto ao acesso às informações. No estudo intitulado "Pesquisa sobre Padrões de Vida" (PPV), realizado pelo Instituto Brasileiro de Geografia e Estatística ([IBGE], 1999), ficou evidenciado o impacto da escolaridade sobre as condições de saúde da população. No entanto, ao analisarem os índices epidemiológicos no Programa Nacional de DST/AIDS (PN-DST/AIDS) do Brasil, Fry, Monteiro, Maio, Bastos e Santos (2007) afirmaram que, no período de 2000 a 2005, a incidência dos casos de AIDS entre indivíduos com educação fundamental reduziu de 39,4\% para $18,6 \%$, embora a variável escolaridade não tenha sido preenchida em $22,4 \%$ dos casos registrados, sendo, portanto, ignorada pelo PN-DST/AIDS. Esses autores afirmaram, ainda, que ocorreu um aumento dos casos de AIDS em pessoas com escolaridade entre ensino médio e superior (de $54,8 \%$ para $76,4 \%$ ), fato que também foi verificado na presente pesquisa, apresentando um expressivo número de participantes com escolaridade variando entre ensino médio incompleto a completo $(42 \%)$.

Outra característica do perfil dos participantes da presente pesquisa diz respeito à interiorização da AIDS, visto que 52\% eram provenientes do interior do Estado da Paraíba, Brasil. O fenômeno da "interiorização da AIDS" vem sendo verificado em outros estudos na literatura (L. M. Barbosa \& Sawyer, 2003; Szwarcwald, Bastos, Esteves, \& Andrade, 2000; UNAIDS: The United Nations Joint Programme on HIV/AIDS, 2002), corroborando os resultados aqui apresentados. Para Szwarcwald et al. (2000, p. 59), "é possível falar em um processo de interiorização da epidemia, à medida que a malha municipal é, gradualmente, afetada pelo processo de expansão da epidemia em curso". Em contraponto, os centros de saúde de referência no atendimento e tratamento da AIDS encontram-se, em sua maior parte, nas capitais ou áreas metropolitanas, o que pode dificultar o acesso aos serviços por parte das pessoas que moram, geograficamente, mais afastadas da capital.

Ressalta-se, ainda, que os participantes apresentaram um tempo médio de diagnóstico de seis anos e, em casos de necessidade de acompanhamento à consulta/internação ou do próprio monitoramento da doença, 63\% afirmaram não possuir cuidador/a; já $30 \%$ dos participantes afirmaram morar sozinhos. Esse resultado é corroborado pelo estudo realizado por Saldanha e Araújo (2006) acerca da AIDS na velhice, demonstrando a pouca participação da família na sobrevida desses idosos e pouca rede de apoio social, bem como o medo do preconceito por parte dos entes queridos.

No tocante à influência das variáveis biodemográficas e clínicas na qualidade de vida dos participantes, os resultados indicaram que houve diferenças estatisticamente significativas entre as médias dos grupos critérios das variáveis biodemográficas e clínicas em relação às médias dos fatores "Autonomia", "Atividades passadas, presentes e futuras", "Morte e morrer" e "Intimidade", o que não ocorreu com os fatores "Funcionamento Sensório" e "Participação Social". Além disso, diferente da pesquisa realizada por Seidl (2005), não foram encontras diferenças nas médias dos participantes quanto ao sexo e quanto à escolaridade, bem como entre as médias dos fatores do WHOQOL-Old e as médias dos grupos critérios das variáveis "local de residência" (interior ou capital), "tipo de religião" e o fato de ter adquirido, ou não, alguma "doença oportunista". Tais resultados podem ser observados na Tabela 1 a seguir.

A partir dos resultados, verifica-se que os participantes soropositivos para o HIV com idades entre 50 a 55 anos apresentaram - em comparação com o grupo de idade mais avançada (e" 56) - menor nível de qualidade de vida nas dimensões "Atividades passadas, presentes e futuras", "Morte e Morrer" e "Intimidade". Assim, as pessoas em idades mais avançadas estavam menos ansiosas em relação à morte e ao morrer. O impacto da AIDS na sua intimidade não foi tão significativo quanto foi para as pessoas com menos idade, também avaliaram as conquistas alcançadas ao longo da vida de modo satisfatório, apresentando-se menos ansiosos em relação a eventos futuros.

A variável "renda" influenciou na avaliação da qualidade de vida dos participantes que possuíam renda familiar de até um salário mínimo, apresentando baixos escores nos fatores "Autonomia", "Atividades passadas, presentes e futuras" e "Intimidade". Nesse sentido, alguns estudos (Cairney \& Arnold, 1998; Fonseca et al., 2000) mencionam a renda como um fator diretamente relacionado às condições de saúde do indivíduo e a sua capacidade funcional, demonstrando a relação existente entre baixa renda e comprometimentos com estado de saúde. No contexto da AIDS, as desigualdades sócio-econômicas têm contribuído de forma significativa para a gravidade da epidemia, apontando para a relação existente entre AIDS e vulnerabilidade social (Ayres et al., 1997), sugerindo-se estudos sobre o impacto dessa relação para a qualidade de vida das pessoas acima de 50 anos.

Considerando-se a variável "situação conjugal" e o fato de morar "só" ou "acompanhado", os resultados indicaram que os participantes deste estudo apresentaram melhor qualidade de vida no fator "Intimidade" quando eram "casados" ou quando moravam "acompanhados". 
Tabela 1

Poder Discriminativo dos Fatores do WHOQOL-Old em Relação às Variáveis Bio-demográficas e Clínicas

\begin{tabular}{|c|c|c|c|c|c|}
\hline Fatores do WHOQOL-Old & & $\mathrm{N}$ & Média & $\mathrm{DP}$ & $\mathrm{p}^{*}$ \\
\hline \multicolumn{6}{|l|}{ Autonomia } \\
\hline \multirow[t]{2}{*}{ Situação Conjugal } & Solteiro & 23 & 66,57 & 10,59 & \multirow{2}{*}{0,021} \\
\hline & Casado/convivente & 20 & 55,31 & 18,16 & \\
\hline \multirow[t]{2}{*}{ Reside } & Só & 13 & 67,78 & 11,36 & \multirow{2}{*}{0,041} \\
\hline & Acompanhado & 30 & 58,54 & 16,37 & \\
\hline \multirow[t]{2}{*}{ Renda Familiar } & $\leq 1$ salários & 32 & 58,20 & 16,14 & \multirow{2}{*}{0,004} \\
\hline & $\geq 2$ salários & 11 & 70,45 & 08,87 & \\
\hline \multicolumn{6}{|l|}{ Atividades passadas, presentes e futuras } \\
\hline \multirow[t]{2}{*}{ Idade } & $50-55$ & 31 & 45,76 & 19,32 & \multirow{2}{*}{0,015} \\
\hline & $\geq 56$ & 12 & 63,54 & 19,18 & \\
\hline \multirow[t]{2}{*}{ Participação em ONG/ grupo de convivência, etc. } & Sim & 17 & 59,55 & 22,86 & \multirow{2}{*}{0,033} \\
\hline & Não & 26 & 44,95 & 17,23 & \\
\hline \multirow[t]{2}{*}{ Renda Familiar } & $\leq 1$ salários & 32 & 45,70 & 18,13 & \multirow{2}{*}{0,016} \\
\hline & $\geq 2$ salários & 11 & 65,34 & 21,53 & \\
\hline \multirow[t]{2}{*}{ Tempo de Diagnóstico } & $\leq 5$ anos & 29 & 43,75 & 16,53 & \multirow{2}{*}{0,004} \\
\hline & $\geq 6$ anos & 14 & 65,17 & 21,47 & \\
\hline \multicolumn{6}{|l|}{ Morte e Morrer } \\
\hline \multirow[t]{2}{*}{ Idade } & $50-55$ & 31 & 26,00 & 31,87 & \multirow{2}{*}{0,000} \\
\hline & $\geq 56$ & 12 & 64,58 & 20,17 & \\
\hline \multirow[t]{2}{*}{ Participação em ONG/ grupo de convivência, etc. } & Sim & 17 & 51,47 & 31,67 & \multirow{2}{*}{0,020} \\
\hline & Não & 26 & 27,16 & 32,06 & \\
\hline \multirow[t]{2}{*}{ Tempo de Diagnóstico } & $\leq 5$ anos & 29 & 25,43 & 30,79 & \multirow{2}{*}{0,001} \\
\hline & $\geq 6$ anos & 14 & 60,26 & 27,47 & \\
\hline \multicolumn{6}{|l|}{ Intimidade } \\
\hline \multirow[t]{2}{*}{ Idade } & $50-55$ & 31 & 40,92 & 27,75 & \multirow{2}{*}{0,034} \\
\hline & $\geq 56$ & 12 & 59,89 & 23,30 & \\
\hline \multirow[t]{2}{*}{ Situação Conjugal } & Solteiro/divorciado & 23 & 31,79 & 20,28 & \multirow{2}{*}{0,000} \\
\hline & Casado/convivente & 20 & 62,81 & 26,16 & \\
\hline Reside & Só & 13 & 26,92 & 15,17 & רחת \\
\hline & Acompanhado & 30 & 54,88 & 27,94 & 0,002 \\
\hline Cuidador & Sim & 16 & 69,92 & 22,26 & $\Omega \rho \cap 0$ \\
\hline & Não & 27 & 32,17 & 20,19 & 0,000 \\
\hline Renda Familiar & $\leq 1$ salários & 32 & 38,47 & 24,18 & $0 \Omega 04$ \\
\hline & $\geq 2$ salários & 11 & 68,75 & 25,92 & 0,004 \\
\hline Tempo de Diagnóstico & $\leq 5$ anos & 29 & 39,00 & 24,92 & \\
\hline & $\geq 6$ anos & 14 & 61,16 & 28,18 & 0,020 \\
\hline
\end{tabular}

Nota. *teste $t ; p<0,05$.

Um fato que se deve considerar é que as pessoas com as quais os participantes afirmaram morar eram, na maioria das vezes, o próprio cônjuge, fato verificado, também, entre os participantes que afirmaram possuir cuidador, o que contribuiu para que esses participantes apresentassem boa qualidade de vida na dimensão "Intimidade". Achados nessa direção foram encontrados por Seidl, Zannon e Tróccoli (2005) no estudo realizado com 241 pessoas soropositivas para o HIV/AIDS. Através da análise de regressão múltipla hierárquica, os pesquisadores verificaram que "viver com parceiro/a" mostrou- se um preditor significativo da dimensão psicossocial da qualidade de vida.

Por outro lado, em comparação com as médias do grupo das pessoas "casadas", com renda de até um salário mínimo e que moravam "acompanhadas", as pessoas "solteiras", com renda maior igual ou maior a dois salários mínimos e que moravam "sós" apresentaram escore médio maior na dimensão "Autonomia”, embora a média do primeiro grupo no fator "Autonomia" tenha sido superior a 50, indicando, também, qualidade de vida satisfatória. 
Esses resultados demonstram a importância do suporte social e afetivo no contexto da AIDS, principalmente a influência desse suporte na avaliação das pessoas sobre sua qualidade de vida, uma vez que o apoio social está relacionado com os cuidados, consolo, suporte, estima ou ajuda que um indivíduo sente receber dos outros (Ogden, 1999). Ademais, a percepção, por parte do indivíduo, de que outras pessoas são sensíveis e receptivas às suas necessidades apresenta-se como fator importante para as estratégias de enfrentamento no lidar com o stress (Aronson, Wilson, \& Akert, 2002), minimizando o impacto negativo da doença e ajudando a pessoa na adaptação a esta (Remor, 1999). Nesse sentido, os resultados indicaram que a participação dos indivíduos em grupo de atividades sociais (ONG's, grupos religiosos, atividades físicas, etc.) contribuiu para uma boa qualidade de vida nas dimensões "Morte e morrer" e "Atividades passadas, presentes e futuras", principalmente no que se refere à ansiedade frente às questões de morte e morrer; os indivíduos sem participação em atividades apresentaram a menor média $(M=27,16)$ nessa dimensão. Dessa forma, é possível que pessoas com ampla rede de apoio social enfrentem a doença com menos sofrimento psíquico e adesão ao tratamento de forma mais permanente.

No tocante à variável clínica "tempo de diagnóstico", os participantes com mais de 5 anos de diagnóstico para o HIV/AIDS apresentaram boa qualidade de vida nos fatores "Atividades presentes, passadas e futuras", "Morte e morrer" e "Intimidade", o que não ocorreu entre os participantes com tempo de diagnóstico de até 5 anos, que apresentaram baixa qualidade de vida nas respectivas dimensões. Nessa direção, no estudo realizado por Paiva, Latorre, Gravato, Lacerda e Initiative-Brazil (2002) com 1.068 mulheres infectadas com HIV/AIDS, os resultados indicaram que, logo após o diagnóstico, houve piora da atividade sexual nessas mulheres, mas havendo, posteriormente, uma adaptação.

Considerando-se a introdução da terapia anti-retroviral, a AIDS transpôs a característica de infecção eminentemente letal, tornando-se uma doença crônica e controlável (Saldanha, 2003), o que contribui para a redução de infecções oportunistas e para uma vida mais produtiva dos indivíduos soropositivos para o HIV. Após longo tempo de diagnóstico, ocorre o que Seidl (2005) chamou de "normalização", ou seja, "a atitude de focalizar a vida dando continuidade aos projetos e ao desempenho de papéis sociais no âmbito da família e do trabalho" (p. 427). No entanto, diferente da normalização definida por Seidl (2005), Saldanha (2003) verificou, em seu estudo com mulheres HIV+, a ocorrência da "naturalização" da AIDS, destacando o caráter temporal da doença e sua relação com as interpretações que os sujeitos fazem no processo de adoecimento. Para essa autora, o "conhecimento" e o "sentido" dado à AIDS são, continuamente, reformulados em decorrência dos processos interativos dos sujeitos, bem como são, frequentemente, confrontados por diferentes diagnósticos e situações construídas pela família, amigos, vizinhos, médicos. Assim, entre as pessoas com maior tempo de conhecimento do diagnóstico, a definição dada à AIDS enquanto doença pode, em alguns casos, ser banalizada, naturalizada ou negada.

\section{Considerações Finais}

Mediante os resultados supracitados, foi possível observar, entre os participantes, a associação entre qualidade de vida, variáveis biodemográficas e clínicas, demonstrando que este construto é uma dimensão da vida humana indissociável de condições objetivas como trabalho, lazer, moradia, dentre outros. No entanto, o caráter multidimensional da qualidade de vida aponta para a necessidade de se considerar, concomitante aos aspectos sócio-econômicos, a influência dos indicadores subjetivos na avaliação que as pessoas fazem sobre sua qualidade de vida. Assim, características próprias de cada indivíduo, como o suporte social, podem influenciar na forma como são percebidos e internalizados os eventos ocorridos. Além disso, a consideração da saúde mental como a avaliação dos transtornos leves - é importante variável clínica para uma melhor avaliação da qualidade de vida. Desse modo, sugere-se a avaliação da relação existente entre o uso dos medicamentos antivirais e a saúde mental de pessoas com mais de 50 anos, o que não foi verificado neste estudo.

O desenvolvimento de estudos na área de qualidade de vida pode subsidiar práticas assistenciais pautadas numa perspectiva ampla do processo saúde-doença, abarcando aspectos socioeconômicos, psicológicos e culturais, importantes nas ações de promoção, prevenção, tratamento e reabilitação em saúde.

\section{Referências}

Araújo, L. F., Coutinho, M. P. L., \& Saldanha, A. A. W. (2005). Análise comparativa das representações sociais da velhice entre idosos de instituições geriátricas e grupos de convivência. Psico, 36(2), 197-204.

Aronson, E., Wilson, T. D., \& Akert, R. M. (2002). Psicologia social (3. ed.). Rio de Janeiro, RJ: LTC.

Arpinelli, F., Visona, G., Bruno, R., Carli, G., \& Apolone, G. (2000). Health-related quality of life in asymptomatic patients with HIV: Evalution of the SF-36 health survey in Italian patients. Pharmacoeconomics, 18, 63-72.

Avis, N. E., Smith, K. W., \& Swislow, L. (1997). Gender differences in quality of life among HIV+ men and women [Abstract 226.6]. National Conference on Women \& HIV, 148, 4-7.

Ayres, J. R. C. M., França, I., Jr., \& Calazans, G. J. (1997). AIDS, vulnerabilidade e prevenção. In II Seminário Saúde Reprodutiva em Tempos de AIDS (pp. 20-37). Rio de Janeiro, RJ: Editora da Universidade do Estado do Rio de Janeiro.

Backman, L., Mantyla, T., \& Herlitz, A. (1990). The optimization of episodic remembering in old age. In P. B. Baltes \& M. M. Baltes (Eds.), Successful aging: Perspectives from the behavioral sciences. Cambridge, MA: Cambridge University. 
Badia, X., Guerra, L., Garcia, M., \& Podzamczer, D. (1999). La evaluación de la calidad de vida en los pacientes con infección por el VIH y sida. Medicina Clinica, 112(19), 739744.

Baltes, P. B., \& Baltes, M. M. (Eds.). (1990). Successful aging: Perspectives from the behavioral sciences. Cambridge, MA: Cambridge University Press,

Barbosa, L. M., \& Sawyer, D. O. (2003). AIDS: A vulnerabilidade social e a evolução da epidemia nos municípios das regiões Nordeste e Sudeste do Brasil. Revista Brasileira de Estudos da População, 20(2), 241-257.

Barbosa, M. T. S., \& Struchiner, C. J. (2003). The estimated magnitude of AIDS in Brazil: A delay correction applied to cases with lost dates. Cadernos de Saúde Pública, 18 , 279-285.

Bastos, F. I., \& Szwarcwald, C. L. (2000). AIDS e pauperização: Principais conceitos e evidências empíricas. Cadernos de Saúde Pública, 16, 65-76.

Brito, A. M., Castilho, E. A., \& Szwarcwald, C. L. (2001). AIDS e infecção pelo HIV no Brasil: Uma epidemia multifacetada. Revista da Sociedade Brasileira de Medicina Tropical, 34(2), 207-217.

Burgoyne, R. W., \& Saunders, D, S. (2001). Quality of life urban Canadian HIV/AIDS clinic outpatients. International Journal of STD \& AIDS, 12(8), 505-512.

Butin, N. G. (2002). Finitude e envelhecimento: Significados da morte no idoso soropositivo. Dissertação de Mestrado não-publicada, Pontifícia Universidade Católica de São Paulo, SP.

Cairney, J., \& Aronold, R. (1998). Socioeconomic position, lifestyle and health among Canadians aged 18 to 64: A multicondition approach. Canadian Journal of Public Health, 89, 208-212.

Canavarro, M. C., Simões, M., Pereira, M., \& Pintassilgo, A. L. (2005). Desenvolvimento dos instrumentos de avaliação da qualidade de vida na infecção VIH da Organização Mundial de Saúde (WHOQOL-HIV; WHOQOL-HIV-BREF) para português de portugal: Apresentação de um projecto. In Anais do VI Congresso Virtual HIV/AIDS - O VIH/SIDA na criança e no idoso. Retrieved July 16, 2007, from http:// www.aidscongress.net/pdf.php?id_comunicacao $=270$

Cloud, G. C., Browne, R., Salooja, N., \& Mclean, K. A. (2003). Newly diagnosed HIV infection in an octogerian: The elderly are not "immune". Age and Ageing, 32(3), 353-354.

Cruz, G. E. C. P. (2005). HIV/AIDS: Um perfil epidemiológico de portadores idosos. Retrieved February 16, 2008, from http://www.portaldoenvelhecimento.net/pforum/aids1.htm

Dias, A., Fonseca, S., Renca, P., \& Silva, E. (2005). A infecção VIH/SIDA na população com +50 anos: Análise estatística da população com +50 anos infectada em Portugal. In Anais do VI Congresso Virtual HIV/AIDS - O VIH/SIDA na criança e no idoso. Retrieved May 02, 2008, from http:// w w w. A I D S c o n g r e s s. n e t / p d f / 50_anos_abstract_233_comunic_266.pdf

Fleck, M. P. A., Chachamovich, E., \& Trentini, C. M. (2006). Development and validation of the Portuguese version of WHOQOL-Old. Revista de Saúde Pública, 40, 785-791.

Fleck, M. P., Leal, O. F., Louzada, S., Xavier, M., Cachamovich, E., Vieira, G., et al. (1999). Desenvolvimento da versão em português do instrumento de avaliação de qualidade de vida da OMS (WHOQOL-100). Revista Brasileira de Psiquiatria, 21, 21-28.
Fonseca, M. G., Francisco, I. B., Derrico, M., Andrade, C. L. T., Travassos, C., \& Szwarcwald, C. L. (2000). AIDS e grau de escolaridade no Brasil: Evolução temporal de 1986 a 1996. Cadernos de Saúde Pública, 16, 77-87.

Fry, P. H., Monteiro, S., Maio, M. C., Bastos, F. I., \& Santos, R. V. (2007). AIDS tem cor ou raça? Interpretação de dados e formulação de políticas de saúde no Brasil. Cadernos de Saúde Pública, 23(3), 497-507.

Instituto Brasileiro de Geografia e Estatística. (1999). Pesquisa sobre padrões de vida 1996-1997 [CD]. Rio de Janeiro, RJ: Autor.

Jakobsson, U., Klevsgard, R., Westergren, A., \& Hallberg, I. R. (2003). Old peoplein pain: A compatative study. Journal of Painand Symptom Management, 26, 625-636.

Ministério da Saúde. (2007). Boletim epidemiológico de DST/ AIDS. Brasília, DF: Autor.

Neri, A. L. (2001). Maturidade e velhice. Campinas, SP: Papirus.

Ogden, J. (1999). Psicologia da saúde. Lisboa, Portugal: Climepsi.

Organização Mundial de Saúde. (1994). Declaração elaborada pelo Grupo de Trabalho da Qualidade de Vida da OMS. Genebra, Suíça: Autor.

Organização Mundial de Saúde. (2005). WHOQOL-Old Manual. Retrieved September 07, 2007, from http:// www.ufrgs.br/psiq/whoqol-old.html

Paiva, V., Latorre, M. R., Gravato, N., Lacerda, R., \& InitiativeBrazil, E. C. (2002). Sexualidade de mulheres vivendo com HIV/AIDS em São Paulo. Cadernos de Saúde Pública, 18(6), 1609-1619.

Remor, E. A. (1999). Abordagem psicológica da AIDS através do enfoque cognitivo-comportamental. Psicologia: Reflexão e Crítica, 12, 89-106.

Ribeiro, P. M. (1997). Telenovelas and the sexuality transition among teenagers in Brazil. Unpublished doctoral dissertation, The University of Texas, Austin, TX

Saldanha, A. A. W. (2003). Vulnerabilidade e construções de enfrentamento da soropositividade ao HIV por mulheres infectadas em relacionamento estável. Tese de Doutorado não-publicada, Faculdade de Filosofia, Ciências e Letras, Universidade de São Paulo, Ribeirão Preto, SP.

Saldanha, A. A. W., \& Araújo, L. F. (2006). A AIDS na terceira idade na perspectiva dos idosos, cuidadores e profissionais de saúde. In VII Congresso Virtual HIV/AIDS - O VIH/SIDA na criança e no idoso. Retrieved August 09, 2008, from www.aidscongress.com/article.php?id_comunicacao $=294$

Saldanha, A. A. W., Figueiredo, M. A. C., \& Coutinho, M. P. L. (2004). Atendimento psicossocial à AIDS: A busca pelas questões subjetivas. Jornal Brasileiro de Doenças Sexualmente Transmissíveis, 16(3), 84-91.

Seidl, E. M. F. (2005). Enfrentamento, aspectos clínicos e sociodemográficos de pessoas vivendo com HIV/AIDS. Psicologia em Estudo, 10(3), 421-429.

Seidl, E. M. F., Zannon, M. L. C., \& Tróccoli, B. T. (2005). Pessoas vivendo com HIV/AIDS: Enfrentamento, suporte social e qualidade de vida. Psicologia: Reflexão e Crítica, 18(2), 188-195

Sorlie, P. D., Backlund, E., \& Keller, J. B. (1995). Mortality by economic, demographic, and social characteristics: The National Longitudinal Mortality Study. American Journal of Public Health, 85, 949-956. 
Souza, Z., \& Leite, J. L. (2002). AIDS e envelhecimento: Reflexões sobre a infecção pelo HIV em indivíduos acima dos 60 anos. In Anais das III e IV Jornadas Científicas do Centro Municipal de Saúde Waldyr Franco. Bangu, RJ: Centro Municipal de Saúde Waldyr Franco. Retrieved from w w w. s a u d e.ri o.r j.gov.br/m e d i a / AIDSENVELHECIMENTO.doc

Szwarcwald, C. L., Bastos, F. I., Esteves, M. A., \& Andrade, C. L. T. (2000). A disseminação da epidemia de AIDS no Brasil, no período de 1987-1996: Uma análise espacial. Cadernos de Saúde Pública, 16, 7-19.

UNAIDS: The United Nations Joint Programme on HIV/AIDS. (2002). Monitoring the Declaration of Commitment on HIVI AIDS: Guidelines on constructions of core indicators. Geneva, Switzerland: Author. 